\title{
Resultados a corto y medio plazo de la técnica de Florida Sleeve en la dilatación de raíz aórtica
}

\author{
Early and medium-term results of the Florida Sleeve technique for aortic root ectasia \\ Edmundo Fajardo-Rodríguez*, Mercedes Castro-Pinto, José López-Menéndez, Javier Miguelena-Hycka, \\ Miren Martín-García, Rafael Muñoz-Pérez y Jorge Rodríguez-Roda \\ Servicio de Cirugía Cardiaca, Hospital Universitario Ramón y Cajal, Madrid, España
}

\begin{abstract}
Resumen
Introducción: La dilatación de la raíz aórtica es una patología frecuente que afecta a pacientes jóvenes y su tratamiento es quirúrgico. Las técnicas quirúrgicas de elección en pacientes jóvenes son las de preservación de la válvula aórtica para evitar el implante de una prótesis valvular. Objetivo: El presente trabajo tiene como objetivo describir la técnica de Florida Sleeve y los resultados de nuestra experiencia. Método: Entre noviembre de 2015 y enero de 2017 fueron intervenidos en nuestro centro tres casos de pacientes con insuficiencia aórtica severa secundaria a anulectasia aórtica en los que se realizó la técnica de Florida Sleeve. Resultados: En los tres casos se obtuvieron excelentes resultados postoperatorios, libres de insuficiencia aórtica residual y de reoperación por cualquier causa, resultados que se mantuvieron a los tres años de seguimiento. Conclusiones: La técnica de Florida Sleeve es una técnica segura, reproducible con una curva de aprendizaje y tiempos quirúrgicos inferiores a las técnicas tradicionales. Los resultados clínicos a medio plazo en términos de morbimortalidad son buenos.
\end{abstract}

Palabras clave: Florida Sleeve. Dilatación de la raíz aórtica. Insuficiencia aórtica.

\begin{abstract}
Introduction: Aortic root dilatation is a frequent disease affecting mostly young patients that often requires surgical repair. Surgical techniques in young patients include aortic valve-sparing procedures to avoid prosthetic valve implant. Objective: The aim of this paper is to describe the results obtained in three patients with aortic root dilatation using the Florida Sleeve technique. Methods: From November 2015 to January 2017, three patients with severe aortic regurgitation due to aortic anuloectasic were intervened applying the Florida Sleeve technique. Results: Excellent postoperative results were obtained in the three cases including freedom of aortic regurgitation and any cause re-operation during three years of follow-up. Conclusion: The Florida Sleeve technique is a safe, reproducible technique with a learning curve and lower surgical times than traditional techniques. The medium-term clinical outcomes in terms of morbidity and mortality are good.
\end{abstract}

Key words: Florida Sleeve. Aortic root dilatation. Aortic regurgitation.

\section{Correspondencia:}

*Edmundo Fajardo-Rodríguez

E-mail: edmundofajardo@ hotmail.com
Disponible en internet: 16-03-2021 Arch Cardiol Mex. 2021;91(4):465-469 www.archivoscardiologia.com

(2020 Instituto Nacional de Cardiología Ignacio Chávez. Publicado por Permanyer. Este es un artículo open access bajo la licencia CC BY-NC-ND (http://creativecommons.org/licenses/by-nc-nd/4.0/).

Fecha de recepción: 02-10-2020 Fecha de aceptación: 17-12-2020 DOI: 10.24875/ACM.20000448 


\section{Introducción}

La dilatación de la raíz aórtica es una patología relativamente frecuente que afecta a un gran porcentaje de pacientes jóvenes. Desde hace aproximadamente 40 años se han descrito técnicas de preservación de la raíz aórtica con buenos resultados a largo plazo $^{1,2}$. Las diferentes técnicas de remodelado y de reimplante de la raíz aórtica se realizan por un número escaso de cirujanos debido a su complejidad y la curva de aprendizaje que suponen ${ }^{3,4}$. En un intento de simplificar el procedimiento se han descrito técnicas alternativas como la de Florida Sleeve, con buenos resultados a medio y largo plazo ${ }^{5-7}$.

El objetivo de este trabajo es analizar los resultados a medio plazo de una serie de tres casos intervenidos de reparación valvular aórtica con la técnica de Florida Sleeve.

\section{Material y métodos}

Entre noviembre de 2015 y enero de 2017 fueron intervenidos en nuestro centro tres pacientes con insuficiencia aórtica severa secundaria a dilatación de la raíz aórtica. Las características clínicas y de imagen se presentan en la tabla 1.

El seguimiento clínico y ecocardiográfico se realizó según el protocolo habitual de nuestro centro para el postoperatorio de plastias valvulares. Se realizó una primera visita con ecocardiografía reglada transtorácica el primer mes posterior a la cirugía, a los seis meses y posteriormente cada año. Los datos correspondientes a las características clínicas y ecocardiografías se obtuvieron de forma retrospectiva de la base de datos electrónica de nuestro centro.

\section{Técnica quirúrgica}

Se utilizó una técnica de circulación extracorpórea (CEC) estándar, con algunos matices dependiendo de cada paciente: en el caso 1 se realizó canulación arterial a nivel de aorta ascendente y venosa bicava, debido a que el paciente tenía que ser intervenido de la válvula mitral y tricúspide de forma concomitante (se realizó una plastia mitral con un anillo protésico de 30 $\mathrm{mm}$ e implante de una neocuerda de Goretex ${ }^{\circledR}$ en el segmento A2 del velo anterior, y una anuloplastia tricúspide con un anillo de $30 \mathrm{~mm}$ ); en los casos 2 y 3 se realizó canulación arterial (aorta ascendente) y venosa (con una cánula atriocava).

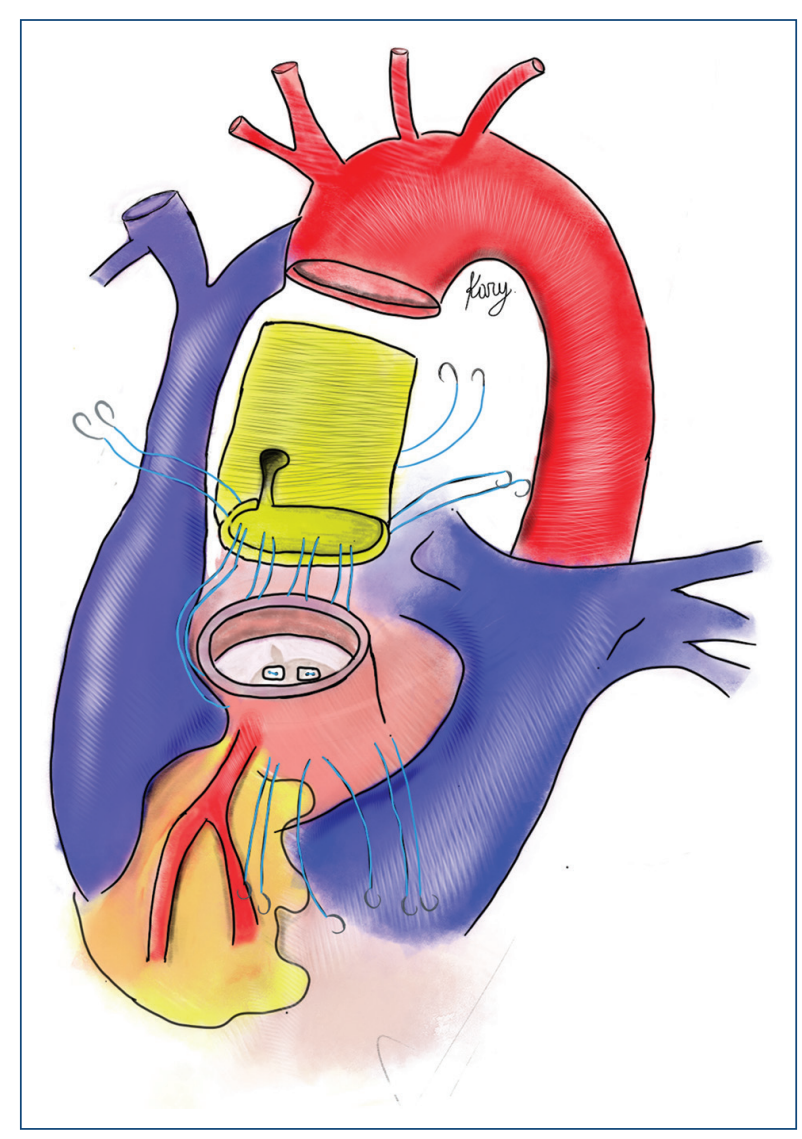

Figura 1. En el tubo de Dacron ${ }^{\circledR}$ se procede a señalar el nivel correspondiente a los ostiums coronarios, se realiza una incisión longitudinal de 1,5 cm a nivel de cada ostium, creando posteriormente una hendidura para encajar la salida de las arterias coronarias. El tubo se ancla mediante suturas de colchonero horizontal por debajo del anillo aórtico (cuatro por cada comisura).

En los tres casos la vía de acceso fue la esternotomía media con una aortotomía trasversa completa superior a la unión sinotubular, se liberó la raíz de la aorta hasta el anillo aórtico incluyendo los segmentos proximales de las arterias coronarias.

La selección del tamaño del tubo protésico se realizó en función del tamaño del anillo aórtico; en los tres casos el anillo midió $27 \mathrm{~mm}$, por lo que se seleccionó un tubo protésico de Dacron ${ }^{\circledR}$ de $32 \mathrm{~mm}$, que sería el tamaño proporcionado de la unión sinotubular para un anillo corregido de $25 \mathrm{~mm}$.

En el tubo de Dacron ${ }^{\circledR}$ se procedió a señalar el nivel correspondiente a los ostiums coronarios, se realizó una incisión longitudinal de $1,5 \mathrm{~cm}$ a nivel de cada ostium desde el extremo proximal del tubo, creando posteriormente una hendidura para encajar la salida de las arterias coronarias (Fig. 1). El tubo se colocó 
Tabla 1. Perfil clínico y características de ecocardiografía y angiotomografía

\begin{tabular}{|l|c|c|c|c|c|c|c|l|}
\hline Caso & Edad & FEVI & NYHA & IAO & Diámetro AoAs & Diámetro AoSin & Diámetro anillo & Bicúspide \\
\hline 1 & 62 & 50 & 2 & 4 & $35 \mathrm{~mm}$ & $47 \mathrm{~mm}$ & $27 \mathrm{~mm}$ & No \\
\hline 2 & 59 & 60 & 1 & 4 & $58 \mathrm{~mm}$ & $47 \mathrm{~mm}$ & $27 \mathrm{~mm}$ & No \\
\hline 3 & 39 & 60 & 2 & 4 & $40 \mathrm{~mm}$ & $45 \mathrm{~mm}$ & $27 \mathrm{~mm}$ & Sí \\
\hline
\end{tabular}

FEVI: fracción de eyección del ventrículo izquierdo; NYHA: grado de disnea según la New York Heart Association; IAO: grado de insuficiencia aórtica; AoAs: aorta ascendente; AoSin: aorta sinusal.

Tabla 2. Datos quirúrgicos

\begin{tabular}{|l|l|c|c|c|}
\hline Caso & Técnica asociada & T ISO & T CEC & IAO residual \\
\hline 1 & PM + PT & 150 & 175 & 1 \\
\hline 2 & No & 85 & 105 & 0 \\
\hline 3 & No & 100 & 115 & 0 \\
\hline
\end{tabular}

PM: plastia mitral; PT: plastia tricúspide; T ISQ: tiempo de isquemia en minutos; T CEC: tiempo de circulación extracorpórea en minutos; IAO: grado de insuficiencia aórtica.

en forma de manga sobre la raíz aórtica y se ancló mediante suturas de colchonero horizontal con poliéster trenzado de 2/0 ubicado por debajo del anillo aórtico en número de 12 (cuatro por cada comisura). Una vez anclado el tubo protésico a la raíz aórtica se procedió a cerrar la hendidura vertical de ambos ostiums coronarios con sutura de polipropileno de $5 / 0$, dejando el diámetro anular de $25 \mathrm{~mm}$ con el objetivo de conseguir una adecuada coaptación de los velos.

El extremo distal del tubo se cortó a nivel de la unión sinotubular y se procedió al cierre hemostático con una sutura continua de polipropileno de $4 / 0$. Finalmente se realizó la anastomosis terminoterminal con el segmento de aorta ascendente (Fig. 2).

En todos los casos se comprobó que la insuficiencia valvular fuera menor de III/IV y la altura de coaptación mayor de $8 \mathrm{~mm}$ mediante ecocardiografía transesofágica intraoperatoria. Los datos relativos a la cirugía se resumen en la tabla 2.

\section{Resultados}

La técnica de Florida Sleeve se realizó en tres pacientes varones, con insuficiencia aórtica severa secundaria a dilatación de la raíz aórtica, en ningún caso la porción sinusal sobrepasaba los $50 \mathrm{~mm}$ de diámetro. Las tres cirugías se practicaron de forma programada, no hubo mortalidad hospitalaria, en la ecocardiografía previa al alta se evidenció una insuficiencia aórtica

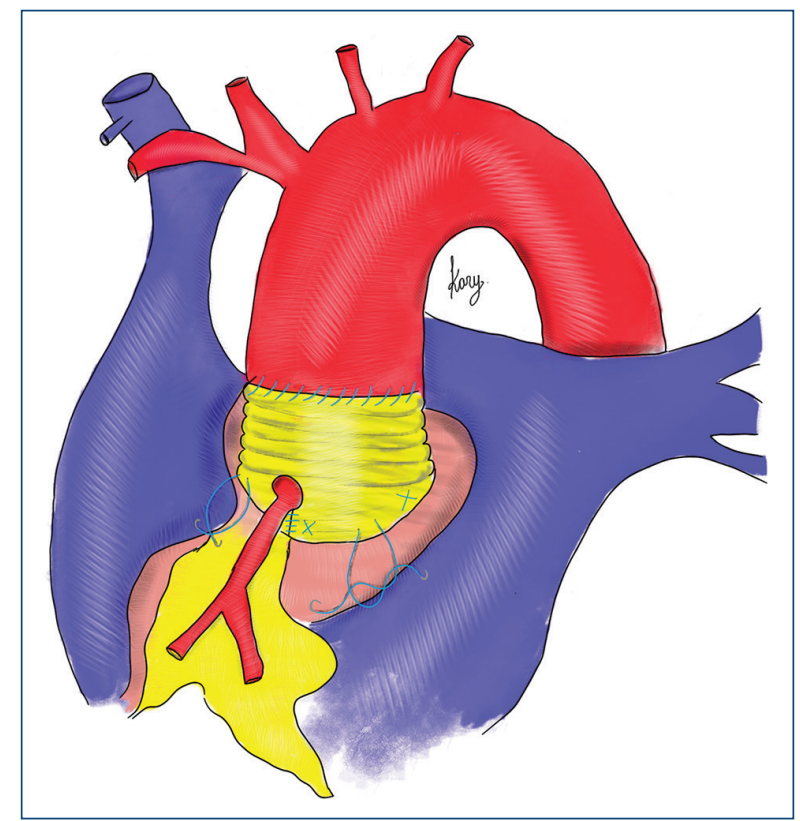

Figura 2. El tubo se coloca en forma de manga sobre la raíz aórtica y una vez anclado el tubo protésico a la raíz se procede a cerrar la hendidura vertical de ambos ostiums coronarios con sutura de polipropileno de 5/0.

residual leve en el caso 1 y en los otros dos pacientes no se evidenció insuficiencia aórtica residual.

El tiempo de CEC en el caso 1 fue 131 minutos, con un tiempo de isquemia de 111 minutos. En los casos 2 y 3 el tiempo medio de isquemia fue de $92.5 \mathrm{~min}$ y de CEC, 110 min.

La estancia media en cuidados intensivos fue de un día y de hospitalización seis días, no hubo accidente cerebrovascular ni necesidad de reoperación por cualquier causa. Ninguno de los tres pacientes requirió trasfusión de hemoderivados, los tres fueron dados de alta en ritmo sinusal y se antiagregaron con $100 \mathrm{mg} /$ día de ácido acetilsalicílico.

En el primer mes postoperatorio los tres pacientes se encontraban en clase funcional I de la NYHA (New York 
Tabla 3. Perfil clínico y características de ecocardiografía a los tres años de seguimiento.

\begin{tabular}{|l|c|c|c|c|c|}
\hline Caso & NYHA & IAO & FEVI & $\begin{array}{c}\text { Diámetro } \\
\text { AoSin }\end{array}$ & $\begin{array}{c}\text { Diámetro } \\
\text { anillo }\end{array}$ \\
\hline 1 & 1 & 1 & 55 & $32 \mathrm{~mm}$ & $25 \mathrm{~mm}$ \\
\hline 2 & 1 & 0 & 60 & $32 \mathrm{~mm}$ & $25 \mathrm{~mm}$ \\
\hline 3 & 1 & 0 & 62 & $32 \mathrm{~mm}$ & $25 \mathrm{~mm}$ \\
\hline
\end{tabular}

FEVI: fracción de eyección del ventrículo izquierdo; NYHA: grado de disnea según la New York Heart Association; IAO: grado de insuficiencia aórtica; AoSin: aorta sinusal.

Heart Association), en ritmo sinusal. La ecocardiografía no evidenció insuficiencia aórtica relevante, la fracción de eyección del ventrículo izquierdo (FEVI) media fue del $59 \%$, sin evidencia de dilatación de la raíz aórtica. Se completó el seguimiento clínico a tres años de todos los pacientes, incluido el estado de supervivencia y la necesidad de reintervención. Ningún paciente desarrolló disección aórtica durante el seguimiento. Los resultados clínicos y ecocardiográficos se mantuvieron durante los tres años de seguimiento. La tabla 3 muestra las características clínicas y ecocardiográficas.

\section{Discusión}

Los aneurismas de aorta ascendente conlleva un alto riesgo de disección o ruptura aórtica en ausencia de tratamiento quirúrgico ${ }^{8}$. En los aneurismas de la raíz aórtica, los pacientes son más jóvenes (30-50 años), con una proporción de sexo de 1:19. La ectasia anuloaórtica puede ser idiopática o asociada con trastornos del tejido conectivo como el síndrome de Marfan, síndrome de Ehler-Danlos o válvula aórtica bicúspi$\mathrm{de}^{10-12}$. Se han desarrollado técnicas quirúrgicas como la de remodelado y reimplante de la raíz aortica para preservar la válvula aórtica ${ }^{1,4}$ y evitar el implante de una prótesis mecánica con la consiguiente anticoagulación que esta conlleva. Debido a la complejidad y curva de aprendizaje de estas técnicas, no constituyen la opción terapéutica de elección en la mayoría de centros $^{5,13}$, siendo la cirugía de Bentall y Bono las más realizada actualmente. La técnica de Florida Sleeve es una alternativa simplificada que precisa menores tiempos de isquemia y CEC en pacientes con una raíz aórtica dilatada que no sobrepase los $50 \mathrm{~mm}$ de diámetro en la porción sinusal, y permite la preservación de la válvula aórtica con buenos resultados a largo plazo $2,4,13,14$.
En nuestro centro se realizó esta técnica en tres pacientes, sin presentar complicaciones intraoperatorias ni en el postoperatorio inmediato, los tiempos de circulación extracorpórea y de parada cardiaca son inferiores a los de la técnica de reimplante de la raíz aórtica (David I), que es la técnica de elección en nuestro centro, aunque por el número limitado de pacientes no se pueden obtener diferencias estadísticamente significativas. El tiempo medio de isquemia y CEC en los últimos 30 paciente en los que se realizó un reimplante valvular tipo David I aislado son de 125 y 159 min respectivamente, por lo que en nuestra escasa experiencia la técnica de Florida Sleeve requiere de unos tiempos sensiblemente inferiores al evitar el reimplante de los tres senos aórticos en el tubo de Dacron ${ }^{\circledR}$. La selección de pacientes se realizó por el criterio del tamaño de la porción sinusal aórtica menor de $50 \mathrm{~mm}$ para evitar una excesiva desproporción entre la prótesis de Dacron $^{\circledR}$ y la aorta nativa.

Los resultados de seguimiento a los tres años han sido satisfactorios, con mejoría de la clase funcional en los tres casos, sin progresión de la insuficiencia aórtica residual y sin deterioro del ventrículo izquierdo evidenciado por ecocardiografía de seguimiento; no se ha registrado la necesidad de reintervención por plastia fallida o por disección de aorta en ninguno de los tres casos.

\section{Conclusiones}

La técnica de Florida Sleeve es una técnica segura, reproducible con una curva de aprendizaje y tiempos quirúrgicos inferiores a las técnicas tradicionales.

Los resultados clínicos a medio plazo en términos de morbimortalidad son buenos. No se evidenció deterioro de la válvula aórtica o dilatación de la raíz aórtica. La función del ventrículo izquierdo se mantiene similar o con una ligera mejoría de la FEVI. Los datos, a pesar de tener un número pequeño de casos, son alentadores y puede ser una alternativa a las técnicas habituales de preservación aórtica en pacientes seleccionados.

\section{Financiamiento}

La presente investigación no ha recibido ninguna ayuda específica de agencias de los sectores público, comercial o sin ánimo de lucro.

\section{Conflicto de intereses}

Los autores declaran no tener conflicto de intereses. 


\section{Responsabilidades éticas}

Protección de personas y animales. Los autores declaran que para esta investigación no se han realizado experimentos en seres humanos ni en animales.

Confidencialidad de los datos. Los autores declaran que han seguido los protocolos de su centro de trabajo sobre la publicación de datos de pacientes.

Derecho a la privacidad y consentimiento informado. Los autores declaran que en este artículo no aparecen datos de pacientes.

\section{Bibliografía}

1. David TE. Aortic valve sparing operations. Ann Thorac Surg. 2002;73(4):1029-30.

2. Aalaei-Andabili SH, Martin TD, Hess PJ, Karimi A, Bavry AA, Arnaoutakis GJ, et al. The Florida Sleeve procedure is durable and improves aortic valve function. AORTA. 2019;07(02):49-55.

3. Minakata K, Schaff HV, Zehr KJ, Dearani JA, Daly RC, Orszulak TA, et al. Is repair of aortic valve regurgitation a safe alternative to valve replacement? J Thorac Cardiovasc Surg. 2004;127(3):645-53.

4. Yacoub MH, Gehle P, Chandrasekaran V, Birks EJ, Child A, Radley-Smith $R$. Late results of a valve-preserving operation in patients with aneu- rysms of the ascending aorta and root. $J$ Thorac Cardiovasc Surg 1998;115(5):1080-90.

5. Saku K, Tobinaga S, Oryoji A, Fukuda T, Zaima $Y$, Saisho H, et al. Florida Sleeve technique for a right sinus of Valsalva aneurysm: a case report. Surg Case Rep. 2019;5(1):125.

6. Hess PJ, Klodell CT, Beaver TM, Martin TD. The Florida Sleeve: A new technique for aortic root remodeling with preservation of the aortic valve and sinuses. Ann Thorac Surg. 2005;80(2):748-50.

7. Aalaei-Andabili SH, Martin T, Hess P, Klodell C, Karimi A, Arnaoutakis G, et al. Florida Sleeve procedure is durable and improves aortic valve function in Marfan syndrome patients. Ann Thorac Surg. 2017;104(3): 834-9.

8. Authors/Task Force members, Windecker S, Kolh P, Alfonso F, Collet J-P, Cremer J, et al. 2014 ESC/EACTS Guidelines on myocardial revascularization: The Task Force on Myocardial Revascularization of the European Society of Cardiology (ESC) and the European Association for Cardio-Thoracic Surgery (EACTS) Developed with the special contribution of the European Association of Percutaneous Cardiovascular Interventions (EAPCI). Eur Heart J. 2014;35(37):2541-619.

9. Nataf $P$. Dilation of the thoracic aorta: medical and surgical management. Heart. 2006;92(9):1345-52

10. Isselbacher EM. Thoracic and abdominal aortic aneurysms. Circulation. 2005;111(6):816-28.

11. Coady MA, Rizzo JA, Goldstein LJ, Elefteriades JA. Natural history, pathogenesis, and etiology of thoracic aortic aneurysms and dissections. Cardiol Clin. 1999;17(4):615-35.

12. Verma S, Siu SC. Aortic dilatation in patients with bicuspid aortic valve. N Engl J Med. 2014;370(20):1920-9.

13. Gamba A, Tasca G, Giannico F, Lobiati E, Skouse D, Galanti A, et al. Early and Medium Term Results of the Sleeve Valve-Sparing Procedure for Aortic Root Ectasia. Ann Thorac Surg. abril de 2015;99(4):1228-33.

14. Tornos P, Sambola A, Permanyer-Miralda G, Evangelista A, Gomez Z, Soler-Soler J. Long-Term Outcome of Surgically Treated Aortic Regurgitation. J Am Coll Cardiol. marzo de 2006;47(5):1012-7. 